\title{
Accessory Os Subtibiale: A case report of misdiagnosed fracture
}

\author{
by V. Madhuri, MS, MCh ${ }^{\natural}$, P.M. Poonnoose, $\mathbf{M S}^{2}$, W. Lurstep, $\mathbf{M S}^{3}$
}

The Foot and Ankle Online Journal 2 (6): 3

Accessory bones of the foot are often misdiagnosed as fractures by trauma surgeons. We report a case of bilateral os subtibiale, which was mistakenly treated as an ankle fracture. The os subtibiale is a rare accessory bone and a variant related to the posterior colliculus of the medial malleolus. This case report with a review of literature emphasizes the importance of being aware of such anomalies.

Key words: Os subtibiale, accessory foot bones, medial malleolus

Published: June, 2009

This is an Open Access article distributed under the terms of the Creative Commons Attribution License. It permits unrestricted use, distribution, and reproduction in any medium, provided the original work is properly cited. (The Foot and Ankle Online Journal (www.faoj.org)

A ccessory bone of the foot are often misdiagnosed as fractures. This report details a case of os subtibiale, a rare accessory bone of the medial malleolus in a patient who presented with an ankle sprain. This patient had previously been misdiagnosed to have a medial malleolar fracture on the opposite ankle.

\section{Case report}

A 25 year-old male presented after twisting the left ankle. Following the injury, the patient is able to walk, bearing some weight on his left foot. On examination there is a swelling over the medial aspect of the left ankle with tenderness over the deltoid ligament of the ankle.

\footnotetext{
Corrseponding address: Professor V Madhuri, Department of Paediatric Orthopaedics,Christian Medical College,Vellore, India, 632004. Telephone : +91- 416-2222102, Fax No. : + 91-416-2232035

Email: madhuriwalter@cmcvellore.ac.in

${ }^{1}$ Madhuri V, MS, MCh (L'pool), Professor and Head, Paediatric Orthopaedics, Department of Paediatric Orthopaedics, Vellore, 632 004, India.

${ }^{2}$ Poonnoose PM, MS Associate Professor, Orthopaedic unit2, Department of Paediatric Orthopaedics, Vellore, 632 004, India.

${ }^{3}$ Lurstep W MS, Registrar Orthopaedic unit 2, Department of Paediatric Orthopaedics, Vellore, 632 004, India.
}

There is no bony tenderness. There is normal and full range of movement of ankle and subtalar joints. Six months prior to this injury the patient was treated with a plaster cast for a fracture of the opposite medial malleolus.

Radiographs of the ankles showed a $2 \times 2 \mathrm{~cm}$ bony ossicle postero-inferior to the medial malleolus. (Fig. 1) The patient is initially diagnosed by the casualty medical officer to have a fracture of the medial malleolus. On review by the Orthopaedic surgeon, it is found that both ankles had similar ossicles below the medial malleolus. The patient is diagnosed to have bilateral os subtibiale and is then treated with compression bandages and anti-inflammatory analgesics. The swelling subsided and the patient is able to walk comfortably within a week. 


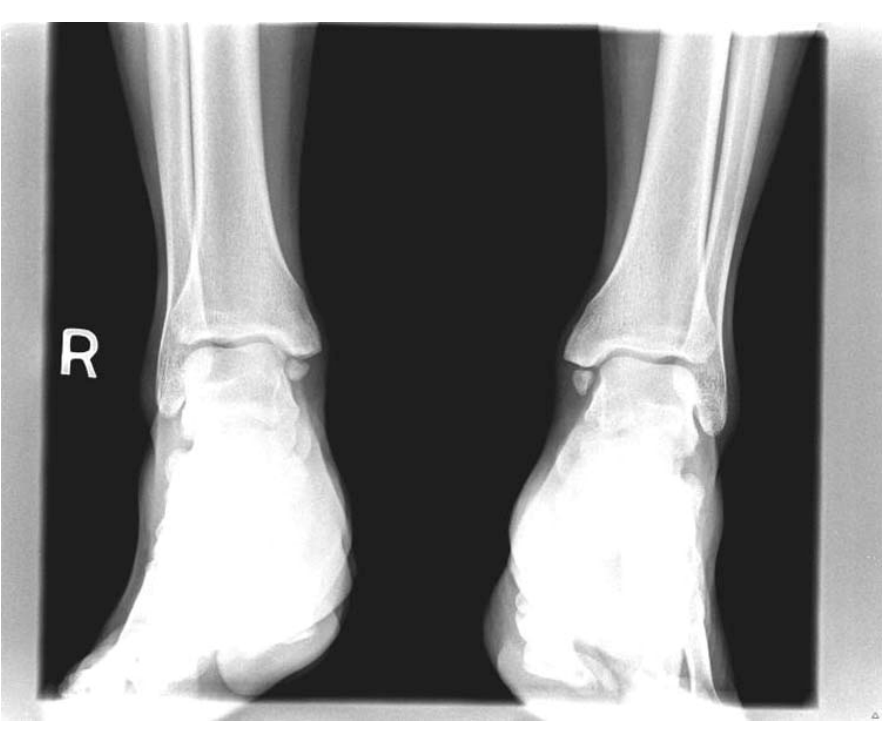

Figure 1 X-ray of the os subtibiale - showing the bony ossicle below the medial malleolus.

\section{Discussion}

Accessory bones of the foot and ankle are often asymptomatic. ${ }^{1,2}$ Most surgeons are familiar with the common accessory bones i.e. the os tibiale externum (the accessory bone of the navicular $(21.3 \%$ ), the os trigonum $(12.7 \%)$ - found behind the posterior process of the talus, and the os peroneum (9\%), the sesamoid bone in the peroneus longus tendon. ${ }^{2}$ Tsuruta, et al., described several other accessory bones like the os subfibulare at the tip of lateral malleolus $(2.1 \%)$, the os supranaviculare, situated above the talonavicular joint $(1 \%)$, and the os supratalare above the neck of the talus $(0.9 \%)^{2}$

There is very little literature available on the accessory bone of the medial malleolus, the os subtibiale. Coral in his series of 700 ankle radiographs, found only one example of the os subtibiale. ${ }^{1}$ It was present in $0.9 \%$ of 3460 radiographs of the foot and ankle studied by Tsuruta T., et al. ${ }^{2}$ In both these radiographic series, the os subtibiale was bilateral, and was found to be located just below the medial malleolus.

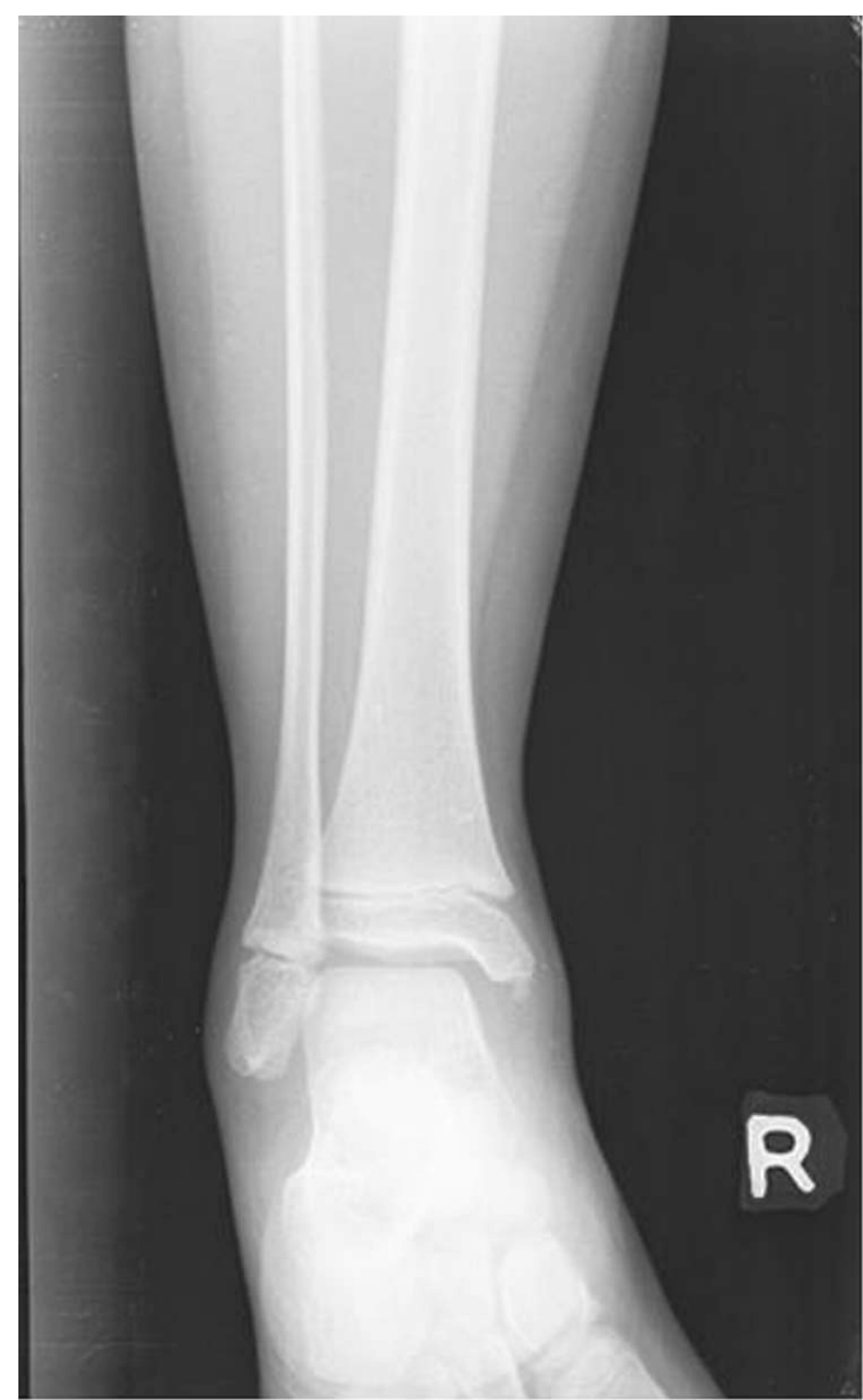

Figure 2 The medial malleolus may occasionally develop from a separate ossification centre at the distal end of the tibia.

The distal epiphyseal centre of the tibia appears in the first year of life and it fuses with the shaft at about age fifteen in females, and seventeen in males. The medial malleolus is generally an extension from the distal epiphysis, and ossifies in the seventh year. ${ }^{3}$ It may occasionally have a separate centre. (Fig.2) An accessory bone is formed when the separate epiphysis of the medial malleolus fails to fuse with the distal tibia. 
The os subtibiale may be mistaken for a malleolar fracture when radiographs are taken following injury to the ankle. ${ }^{4}$ The patient described by Coral had an unnecessary surgery performed after misinterpretation of the X-ray. ${ }^{4}$ When a patient presents with trauma to the ankle, there are some features that help differentiate the os subtibiale from a fracture. First, the edges of the accessory bone are smooth and rounded on the X-ray - unlike a fractured malleolus, which is usually irregular. Secondly, the os subtibiale is often bilateral and is present just below the region of the medial malleolus. ${ }^{1}$ Third, the site of maximum tenderness is usually not directly over the medial malleolus - but below it, over the ligaments. When the os subtibiale is associated with a ligamentous injury, conservative treatment is usually adequate. This patient had previously been treated with a plaster cast as he had been misdiagnosed to have a right medial malleolar fracture.

This case report demonstrates the ease with which os subtibiale can be mistaken for a fracture. An increased awareness and knowledge of such accessory bones of the foot along with clinical and radiological correlation is needed to make a correct diagnosis.

\section{References}

1. Coral A: The radiology of skeletal elements in the subtibial region: incidence and significance. Skeletal Radiol 16 (4): 298 303, 1987.

2. Tsuruta T, Shiokawa Y, Kato A, Matsumoto T, Yamazoe Y, Oike T, Sugiyama T, Saito M: Radiological study of the accessory skeletal elements in the foot and ankle. Nippon Seikeigeka Gakkai Zasshi 55(4): 357 - 370, 1981.

3. Soames RW: Skeletal System. In: Peter L Williams (Editor). Gray's Anatomy. 38 $8^{\text {th }}$ Edition. Churchill Livingstone, London, 697, 1995.

4. Coral A: Os subtibiale mistaken for a recent fracture. Br Med J (Clin Res Ed) 292 (6535): 1571 - 1572, 1986. 